\title{
FUNCTIONS IN BLOCH-TYPE SPACES AND THEIR MODULI
}

\author{
Konstantin M. Dyakonov
}

ICREA, BGSMath and Universitat de Barcelona, Departament de Matemàtiques i Informàtica Gran Via 585, E-08007 Barcelona, Spain; konstantin.dyakonov@icrea.cat

\section{In memory of Victor Petrovich Havin}

\begin{abstract}
Given a suitably regular nonnegative function $\omega$ on $(0,1]$, let $\mathcal{B}_{\omega}$ denote the space of all holomorphic functions $f$ on the unit ball $\mathbf{B}_{n}$ of $\mathbf{C}^{n}$ that satisfy

$$
|\nabla f(z)| \leq C \frac{\omega(1-|z|)}{1-|z|}, \quad z \in \mathbf{B}_{n}
$$

with some fixed $C=C_{f}>0$. We obtain a new characterization of $\mathcal{B}_{\omega}$ functions in terms of their moduli.
\end{abstract}

\section{Introduction and results}

Let $\mathcal{H}\left(\mathbf{B}_{n}\right)$ denote the space of holomorphic functions on the ball

$$
\mathbf{B}_{n}:=\left\{z \in \mathbf{C}^{n}:|z|<1\right\}
$$

(we write $|\cdot|$ for the usual Euclidean norm on $\mathbf{C}^{n}$ ). Talking about subclasses of $\mathcal{H}\left(\mathbf{B}_{n}\right)$, or just about function classes in general, we may single out two large families of spaces. First, there are growth spaces defined by imposing an explicit size condition, either integral or uniform, on the function's modulus. A growth space $X \subset \mathcal{H}\left(\mathbf{B}_{n}\right)$ will typically have the "lattice property": whenever $f \in X$ and $g \in \mathcal{H}\left(\mathbf{B}_{n}\right)$ satisfy $|f| \geq|g|$ on $\mathbf{B}_{n}$, it follows that $g \in X$. This family contains the classical Hardy and Bergman spaces, various weighted $H^{\infty}$ spaces involving specific majorants on the modulus, etc. Secondly, there are smoothness spaces defined in terms of derivatives and/or differences that are built from the function itself (rather than from its modulus). Among the representatives of the latter family are the Lipschitz, Besov and Sobolev spaces, to mention a few.

Rather surprisingly, it turns out that a number of (holomorphic) smoothness spaces nevertheless admit a fairly explicit description in terms of the moduli of their members. The conditions that arise should, of course, govern the oscillations of the function's modulus, not just its growth rate. For Lipschitz spaces, several such characterizations were obtained by the author in [3] for the case of the disk $\mathbf{D}:=\mathbf{B}_{1}$. They were subsequently extended in $[6]$ to $\mathbf{B}_{n}$, and in fact to more general domains in $\mathbf{C}^{n}$. We also cite [4] in connection with holomorphic Besov spaces on D.

doi:10.5186/aasfm.2016.4143

2010 Mathematics Subject Classification: Primary 30H05, 32A37, 32A38, 46J10.

Key words: Holomorphic functions, Bloch-type spaces, Lipschitz spaces.

Supported in part by grants MTM2011-27932-C02-01, MTM2014-51834-P from El Ministerio de Economía y Competitividad (Spain) and grant 2014-SGR-289 from AGAUR (Generalitat de Catalunya). 
The purpose of this note is to provide a similar characterization for certain "Blochtype" spaces that result from a growth restriction on the gradient

$$
\nabla f=\left(\partial_{1} f, \ldots, \partial_{n} f\right)
$$

of a function $f \in \mathcal{H}\left(\mathbf{B}_{n}\right)$; here $\partial_{j}$ stands for the partial differentiation operator $\frac{\partial}{\partial z_{j}}$. More precisely, given a (reasonably nice) positive function $\omega$ on the interval $(0,1]$, the associated Bloch-type space $\mathcal{B}_{\omega}=\mathcal{B}_{\omega}\left(\mathbf{B}_{n}\right)$ consists, by definition, of the functions $f \in \mathcal{H}\left(\mathbf{B}_{n}\right)$ that obey the condition

$$
|\nabla f(z)| \leq C_{f} \frac{\omega(1-|z|)}{1-|z|}, \quad z \in \mathbf{B}_{n}
$$

with some fixed constant $C_{f}>0$ on the right.

To be more specific about the class of $\omega$ 's we have in mind, we now introduce the appropriate terminology. We say that a function $\omega:(0,1] \rightarrow(0, \infty)$ is moderate if there is a constant $C>0$ with the following property: whenever $a \in(0,1]$ and $b \in(0,1]$ satisfy

$$
\frac{1}{2} \leq \frac{a}{b} \leq 2
$$

we have

$$
\frac{1}{C} \leq \frac{\omega(a)}{\omega(b)} \leq C
$$

In particular, if $\omega$ is a nondecreasing (resp., nonincreasing) positive function such that the ratio $\omega(2 t) / \omega(t)$ is bounded above (resp., below) for $0<t \leq \frac{1}{2}$, then $\omega$ is moderate.

If $\omega(t)$ tends to 0 fast enough as $t \rightarrow 0^{+}$, so that $\omega(t)=o(t)$, then no nonconstant function is in $\mathcal{B}_{\omega}$. (Obviously, this is not the case we are interested in.) By contrast, $\mathcal{B}_{\omega}$ is sure to be nontrivial once we assume that the function $t \mapsto \omega(t) / t$ is nonincreasing. Now, if $\omega$ is a nondecreasing function with the latter property, and if $\omega$ is "not too slow" near 0 in the sense that

$$
\int_{0}^{\delta} \frac{\omega(t)}{t} d t \leq \mathrm{const} \cdot \omega(\delta), \quad 0<\delta<1
$$

(such $\omega$ 's are called fast majorants in [6]), then $\mathcal{B}_{\omega}$ becomes the holomorphic Lipschitz space $\Lambda_{\omega}$ associated with $\omega$; its members are precisely the functions whose modulus of continuity is dominated by $\omega$. The special case $\omega(t)=t^{\alpha}(0<\alpha \leq 1)$ corresponds to the classical Lipschitz space of order $\alpha$.

When $\omega(t) \equiv 1$, the space $\mathcal{B}_{\omega}$ reduces to the usual Bloch space $\mathcal{B}$. The little Bloch space $\mathcal{B}_{0}$, formed by the functions $f \in \mathcal{H}\left(\mathbf{B}_{n}\right)$ with

$$
|\nabla f(z)| \cdot(1-|z|) \rightarrow 0 \quad \text { as } \quad|z| \rightarrow 1^{-},
$$

can be written as $\bigcup_{\omega} \mathcal{B}_{\omega}$, where $\omega$ runs through the collection of all (moderate) functions with $\lim _{t \rightarrow 0^{+}} \omega(t)=0$. On the other hand, if $\omega$ satisfies $\lim _{t \rightarrow 0^{+}} \omega(t)=\infty$, then the associated $\mathcal{B}_{\omega}$ space is clearly larger than $\mathcal{B}$ and possesses a kind of "negative order" smoothness. Furthermore, if $\omega(t)$ happens to grow rapidly enough as $t \rightarrow 0^{+}$, then $\mathcal{B}_{\omega}$ becomes a growth space, meaning that it can be described by a size condition on $|f|$. For instance, letting $\omega(t)=t^{-\beta}$ with some $\beta>0$, one may rewrite (1.1) in the simpler form

$$
|f(z)| \leq \frac{\text { const }}{(1-|z|)^{\beta}}, \quad z \in \mathbf{B}_{n}
$$


However, the case of a milder (say, logarithmic) growth rate of $\omega$ near 0 is more delicate: the resulting $\mathcal{B}_{\omega}$ space is then closer in nature to $\mathcal{B}$, and it is no longer describable in terms of a growth estimate on $|f(z)|$ as $|z| \rightarrow 1^{-}$.

Finally, a bit of notation will be needed. For a point $z \in \mathbf{B}_{n}$, we put

$$
d_{z}:=1-|z|
$$

and let $B_{z}$ denote the (Euclidean) open ball with center $z$ and radius $d_{z} / 2$. Also, with a function $f \in \mathcal{H}\left(\mathbf{B}_{n}\right)$ and a point $z \in \mathbf{B}_{n}$ we associate the quantity

$$
M_{f}(z):=\sup \left\{|f(w)|: w \in B_{z}\right\} .
$$

Next, we introduce the zero set

$$
\mathcal{Z}_{f}:=\left\{\zeta \in \mathbf{B}_{n}: f(\zeta)=0\right\}
$$

and define

$$
E_{f}:=\left\{z \in \mathbf{B}_{n}: B_{z} \cap \mathcal{Z}_{f} \neq \emptyset\right\} .
$$

Thus $E_{f}$ can be viewed as a neighborhood of $\mathcal{Z}_{f}$; and if $f$ happens to be zero-free (i.e., $\mathcal{Z}_{f}=\emptyset$ ), then we also have $E_{f}=\emptyset$.

Our main result is as follows. When stating it, and later on, we write $E:=E_{f}$ and $E^{c}:=\mathbf{B}_{n} \backslash E_{f}$.

Theorem 1.1. Given $f \in \mathcal{H}\left(\mathbf{B}_{n}\right)$ and a moderate function $\omega:(0,1] \rightarrow(0, \infty)$, the following are equivalent.

(i) $f \in \mathcal{B}_{\omega}$.

(ii) There is a constant $C_{1}>0$ such that

$$
\sup \left\{\left|f\left(z_{1}\right)\right|-\left|f\left(z_{2}\right)\right|: z_{1}, z_{2} \in B_{z}\right\} \leq C_{1} \cdot \omega\left(d_{z}\right), \quad z \in \mathbf{B}_{n} .
$$

(iii) There is a constant $C_{2}>0$ such that

$$
\chi_{E}(z) \cdot M_{f}(z)+|f(z)| \log \frac{M_{f}(z)}{|f(z)|} \leq C_{2} \cdot \omega\left(d_{z}\right), \quad z \in \mathbf{B}_{n}
$$

(the second term on the left being understood as 0 if $z \in \mathcal{Z}_{f}$ ).

(iv) There is a constant $C_{3}>0$ such that

$$
\chi_{E}(z) \cdot M_{f}(z)+\chi_{E^{c}}(z) \cdot|f(z)| \log \frac{M_{f}(z)}{|f(z)|} \leq C_{3} \cdot \omega\left(d_{z}\right), \quad z \in \mathbf{B}_{n} .
$$

We emphasize that conditions (ii), (iii) and (iv) above are indeed expressed in terms of $|f|$ alone. Conditions (iii) and (iv) are new even in the Lipschitz case, while (ii) is perhaps not too far from what was known previously. In fact, the implications (i) $\Longrightarrow$ (ii) $\Longrightarrow$ (iii) $\Longrightarrow$ (iv) are either elementary or trivial (or both), so the main effort consists in verifying that the last - and formally weakest - condition on $|f|$ is actually sufficient to ensure that $f \in \mathcal{B}_{\omega}$. In a sense, (iv) seems to be the weakest possible condition on the modulus that does the job. We also remark that conditions (iii) and (iv), which invlove "logarithmic oscillations", are usually easier to check than (ii) or similar Lipschitz-type conditions on $|f|$, such as those that arose in $[3,6]$ in the $\Lambda_{\omega}$ setting.

Given a nonvanishing function $f \in \mathcal{H}\left(\mathbf{B}_{n}\right)$, we have $E=\emptyset$ and $E^{c}=\mathbf{B}_{n}$, in which case conditions (iii) and (iv) become the same. Each of these reduces to saying that

$$
|f(z)| \log \frac{M_{f}(z)}{|f(z)|} \leq \text { const } \cdot \omega\left(d_{z}\right), \quad z \in \mathbf{B}_{n} .
$$


To see a consequence of this criterion, let us now consider a nonvanishing holomorphic function $F$ on the unit disk $\mathbf{D}:=\mathbf{B}_{1}$ in $\mathbf{C}$. (In what follows, we also use the notation $\mathbf{T}:=\partial \mathbf{D}$ for the unit circle, and $m$ for the normalized arclength measure on T.) Assume, in addition, that $F$ lies in some Hardy space $H^{p}=H^{p}(\mathbf{D})$ with $0<p \leq \infty$. It is well known (see, e.g., [7, Chapter II]) that, except for a constant factor of modulus 1 , such a function is necessarily of the form

$$
F(z)=\exp \left(\int_{\mathbf{T}} \frac{\zeta+z}{\zeta-z} d \nu(\zeta)\right), \quad z \in \mathbf{D},
$$

$\nu$ being a signed measure on $\mathbf{T}$. This measure can further be written as

$$
d \nu=\log \psi d m-d \mu_{s}
$$

where $\psi$ is a nonnegative function satisfying

$$
\psi \in L^{p}(\mathbf{T}, m) \quad \text { and } \quad \log \psi \in L^{1}(\mathbf{T}, m)
$$

(in fact, $\psi(\zeta)=\lim _{r \rightarrow 1^{-}}|F(r \zeta)|$ for $m$-almost every $\zeta \in \mathbf{T}$ ), while $\mu_{s}$ is a finite positive measure on $\mathbf{T}$ singular with respect to $m$. When $\mu_{s}=0, F$ becomes the outer function with modulus $\psi$, whereas taking $\psi \equiv 1$ one gets the singular inner function associated with $\mu_{s}$; again, we refer to [7, Chapter II] for these matters.

It is straightforward to verify that

$$
\log |F(z)|=\int_{\mathbf{T}} \frac{1-|z|^{2}}{|\zeta-z|^{2}} d \nu(\zeta)=: \mathcal{P} \nu(z)
$$

for $z \in \mathbf{D}$; here $\mathcal{P}$ stands for the Poisson integral operator. Therefore, the next result comes out readily upon applying (1.3) to $F$ in place of $f$. The space $\mathcal{B}_{\omega}$ in the statement below should be understood as $\mathcal{B}_{\omega}(\mathbf{D})$. Likewise, the symbols $d_{z}$ and $B_{z}$ will have the same meaning as before, but restricted to dimension $n=1$.

Theorem 1.2. Suppose $F$ is defined by (1.4), with $\nu$ as in (1.5). Given $p \in$ $(0, \infty]$ and a moderate function $\omega:(0,1] \rightarrow(0, \infty)$, the following are equivalent.

(a) $F \in \mathcal{B}_{\omega} \cap H^{p}$.

(b) $\psi$ satisfies (1.6), and there is a constant $C>0$ such that

$$
\exp (\mathcal{P} \nu(z)) \cdot[\mathcal{P} \nu(w)-\mathcal{P} \nu(z)] \leq C \cdot \omega\left(d_{z}\right)
$$

whenever $z \in \mathbf{D}$ and $w \in B_{z}$.

In particular, letting $\mu_{s}=0$, one arrives at a criterion for an outer function to be in $\mathcal{B}_{\omega} \cap H^{p}$. In the Lipschitz case, when $\mathcal{B}_{\omega}\left(=\mathcal{B}_{\omega} \cap H^{p}\right)=\Lambda_{\omega}$, the result is also new and supplements previous characterizations of the outer functions in $\Lambda_{\omega}=\Lambda_{\omega}(\mathbf{D})$ that were found by Shirokov $[10,11]$ and by the author $[3,5]$. On the other hand, letting $\psi \equiv 1$ (and $p=\infty$, say), one obtains a description of the singular inner functions in $\mathcal{B}_{\omega}$; the class of such singular inner functions is nontrivial when $\omega(t)$ tends to 0 slowly enough as $t \rightarrow 0^{+}$.

One might also consider the "little oh" analogues of the $\mathcal{B}_{\omega}$ spaces and come up with the corresponding "little oh" version of the theorem above. We restrict ourselves to mentioning the case of $\mathcal{B}_{0} \cap H^{\infty}$, where $\mathcal{B}_{0}=\mathcal{B}_{0}(\mathbf{D})$ is the little Bloch space defined by condition (1.2), this time with $\left|f^{\prime}(z)\right|$ in place of $|\nabla f(z)|$.

Proposition 1.3. Suppose $F$ is a function of the form (1.4), with $\nu$ as in (1.5). In order that $F \in \mathcal{B}_{0} \cap H^{\infty}$, it is necessary and sufficient that $\psi \in L^{\infty}(\mathbf{T}, m)$ and

$$
\sup \left\{\exp (\mathcal{P} \nu(z)) \cdot[\mathcal{P} \nu(w)-\mathcal{P} \nu(z)]: w \in B_{z}\right\} \rightarrow 0
$$

as $|z| \rightarrow 1^{-}$. 
It might be interesting to compare this with Bishop's characterization of $\mathcal{B}_{0} \cap H^{\infty}$, as given in [2].

Postponing the proof of Theorem 1.1 to the last section, we shall begin by establishing a preliminary result (see Section 2 below), namely a certain Schwarz-Pick type lemma, to lean upon. The idea of using this kind of technique for similar purposes goes back to Pavlović's paper [8], where the classical Schwarz(-Pick) lemma was employed to give a simple proof of the author's earlier result from [3] on the moduli of holomorphic Lipschitz functions. Here, we use a refined version of the Schwarz-Pick inequality that is valid for nonvanishing functions only (even though the function $f$ of Theorem 1.1 may have zeros). This allows us to arrive at the required estimate on $|\nabla f(z)|$ for $z \notin E_{f}$, while the case of $z \in E_{f}$ is treated separately; see the proof of the (iv) $\Longrightarrow$ (i) part in Section 3 .

\section{A Schwarz-Pick type lemma for nonvanishing functions}

The familiar Schwarz-Pick lemma (see, e.g., [7, Chapter I]) tells us that if $g$ is a holomorphic self-map of the unit disk $\mathbf{D}$ (in $\mathbf{C}$ ), then

$$
\left|g^{\prime}(z)\right| \leq \frac{1-|g(z)|^{2}}{1-|z|^{2}}
$$

for all $z \in \mathbf{D}$. See also [9, Chapter 8] for extensions of this to $\mathbf{B}_{n}$ with $n>1$. Now, it turns out that if $g$ happens to be zero-free, then a better estimate is possible; the refinement is given (in the $\mathbf{B}_{n}$ setting) by Lemma 2.1 below. In the case of $\mathbf{D}$, the result is essentially known. For instance, it can be deduced from the generalized Schwarz-Pick lemma due to Ahlfors; see Theorem 1-7 in [1, Chapter 1]. However, since the required version-which should also work for $\mathbf{B}_{n}$ - does not seem to be readily available in the literature, we have chosen to provide a complete self-contained proof thereof.

Lemma 2.1. Suppose $g \in \mathcal{H}\left(\mathbf{B}_{n}\right)$ is a function satisfying $0<|g(z)| \leq 1$ for all $z \in \mathbf{B}_{n}$. Then

$$
|\nabla g(z)| \leq \frac{2}{1-|z|^{2}}|g(z)| \log \frac{1}{|g(z)|}, \quad z \in \mathbf{B}_{n}
$$

In particular,

$$
|\nabla g(0)| \leq 2|g(0)| \log \frac{1}{|g(0)|}
$$

Proof. First let us consider the case $n=1$. Thus, $g$ is currently supposed to be a holomorphic function on the disk $\mathbf{D}:=\mathbf{B}_{1}$ satisfying $0<|g| \leq 1$ there. We may furthermore assume that $g$ is an outer function. (Otherwise, replace $g$ by $g_{r}$ with $0<r<1$, where $g_{r}(z):=g(r z)$, and then let $r \rightarrow 1^{-}$.) This last assumption means that the (nonpositive) harmonic function $h:=\log |g|$ is the Poisson integral of its boundary values:

$$
h(z)=\int_{\mathbf{T}} \frac{1-|z|^{2}}{|\zeta-z|^{2}} h(\zeta) d m(\zeta)=: \mathcal{P}\left(\left.h\right|_{\mathbf{T}}\right)(z), \quad z \in \mathbf{D}
$$

while $g$ itself is of the form

$$
g(z)=\exp \left(\int_{\mathbf{T}} \frac{\zeta+z}{\zeta-z} h(\zeta) d m(\zeta)\right), \quad z \in \mathbf{D} .
$$


Differentiating (2.4) gives

$$
g^{\prime}(z)=g(z) \cdot U(z)
$$

where

$$
U(z):=\int_{\mathbf{T}} \frac{2 \zeta}{(\zeta-z)^{2}} h(\zeta) d m(\zeta)
$$

Now, since $0 \leq-h=|h|$ almost everywhere on $\mathbf{T}$, we have

$$
|U(z)| \leq-\int_{\mathbf{T}} \frac{2}{|\zeta-z|^{2}} h(\zeta) d m(\zeta)=-\frac{2}{1-|z|^{2}} \mathcal{P}\left(\left.h\right|_{\mathbf{T}}\right)(z)=-\frac{2}{1-|z|^{2}} h(z),
$$

where the last step relies on (2.3). In conjunction with (2.5), this yields

$$
\left|g^{\prime}(z)\right|=|g(z)| \cdot|U(z)| \leq-\frac{2|g(z)|}{1-|z|^{2}} h(z)=\frac{2|g(z)|}{1-|z|^{2}} \log \frac{1}{|g(z)|} .
$$

We have thereby established (2.1), and in particular (2.2), in dimension $n=1$.

Our next step is to prove $(2.2)$ in the case $n>1$. Assuming that $\nabla g(0) \neq 0$ (otherwise the inequality is trivial), we consider the unit vector

and put

$$
\zeta=\nabla g(0) /|\nabla g(0)|=\frac{1}{|\nabla g(0)|}\left(\partial_{1} g(0), \ldots, \partial_{n} g(0)\right)
$$

$$
G(w):=g(w \bar{\zeta}), \quad w \in \mathbf{D}
$$

Because $G$ is a holomorphic function on $\mathbf{D}$ with $0<|G| \leq 1$ and

$$
G^{\prime}(0)=\langle\nabla g(0), \zeta\rangle=|\nabla g(0)|,
$$

where $\langle\cdot, \cdot\rangle$ denotes the usual inner product in $\mathbf{C}^{n}$, the (already known) inequality

$$
\left|G^{\prime}(0)\right| \leq 2|G(0)| \log \frac{1}{|G(0)|}
$$

reduces to (2.2); the latter is thus established for every $n$.

Finally, to prove (2.1) in full generality, we fix a nonzero point $a \in \mathbf{B}_{n}$ and consider the automorphism $\varphi_{a}$ of $\mathbf{B}_{n}$ that interchanges $a$ and 0 . This is given by

$$
\varphi_{a}(z)=\frac{a-P_{a} z-\left(1-|a|^{2}\right)^{1 / 2} Q_{a} z}{1-\langle z, a\rangle}, \quad z \in \mathbf{B}_{n},
$$

where $P_{a}$ is the orthogonal projection of $\mathbf{C}^{n}$ onto the one-dimensional subspace spanned by $a$, and $Q_{a}=I-P_{a}$ is the complementary projection. Then we define

$$
F(z):=\left(g \circ \varphi_{a}\right)(z), \quad z \in \mathbf{B}_{n},
$$

so that $F$ is a holomorphic function on $\mathbf{B}_{n}$ satisfying $0<|F| \leq 1$ there. An application of (2.2), with $F$ in place of $g$, yields

$$
|\nabla F(0)| \leq 2|F(0)| \log \frac{1}{|F(0)|} .
$$

Differentiating (2.6) and bearing in mind that $\varphi_{a} \circ \varphi_{a}$ is the identity map, we find that

$$
\nabla g(a)=\nabla F(0) \cdot\left(\varphi_{a}^{\prime}(0)\right)^{-1}=\nabla F(0) \cdot \varphi_{a}^{\prime}(a),
$$

where the gradients are interpreted as row vectors, while $\varphi_{a}^{\prime}$ stands for the appropriate Jacobian matrix. The formula

$$
\varphi_{a}^{\prime}(a)=-\left(1-|a|^{2}\right)^{-1} P_{a}-\left(1-|a|^{2}\right)^{-1 / 2} Q_{a}
$$


(see [9, Section 2.2]) implies readily that $\left\|\varphi_{a}^{\prime}(a)\right\|$, the operator norm of the matrix $\varphi_{a}^{\prime}(a)$, is bounded by $\left(1-|a|^{2}\right)^{-1}$. It now follows from $(2.8)$ that

$$
|\nabla g(a)| \leq \frac{1}{1-|a|^{2}} \cdot|\nabla F(0)|
$$

Finally, we notice that $F(0)=g(a)$ and combine (2.7) with (2.9) to obtain

$$
|\nabla g(a)| \leq \frac{2}{1-|a|^{2}}|g(a)| \log \frac{1}{|g(a)|}
$$

This is precisely (2.1), with $a$ in place of $z$, and we are done.

\section{Proof of Theorem 1.1}

(i) $\Longrightarrow$ (ii). Fix $z \in \mathbf{B}_{n}$ and let $z_{1}, z_{2} \in B_{z}$. Clearly,

$$
\left|f\left(z_{1}\right)\right|-\left|f\left(z_{2}\right)\right| \leq\left|f\left(z_{1}\right)-f\left(z_{2}\right)\right| \leq \int_{\left[z_{1}, z_{2}\right]}|\nabla f(\zeta)||d \zeta|,
$$

where $\left[z_{1}, z_{2}\right]$ denotes the segment with endpoints $z_{1}$ and $z_{2}$. Since $f \in \mathcal{B}_{\omega}$, we have

$$
|\nabla f(\zeta)| \leq C \frac{\omega\left(d_{\zeta}\right)}{d_{\zeta}} \leq C_{1} \frac{\omega\left(d_{z}\right)}{d_{z}}, \quad \zeta \in\left[z_{1}, z_{2}\right]
$$

where $C$ and $C_{1}$ are suitable constants. (The last inequality in (3.2) is due to the fact that $\frac{1}{2} d_{z} \leq d_{\zeta} \leq \frac{3}{2} d_{z}$ for $\zeta \in B_{z}$, combined with the hypothesis on $\omega$.) Using (3.2) to estimate the integral in (3.1), while noting that the length of $\left[z_{1}, z_{2}\right]$ is at most $d_{z}$, we obtain

which proves (ii).

$$
\left|f\left(z_{1}\right)\right|-\left|f\left(z_{2}\right)\right| \leq C_{1} \omega\left(d_{z}\right)
$$

(ii) $\Longrightarrow$ (iii). Let $z \in E\left(=E_{f}\right)$, so that $B_{z}$ contains a point $z_{0}$ with $f\left(z_{0}\right)=0$. For $w \in B_{z}$, (ii) yields

$$
|f(w)|=|f(w)|-\left|f\left(z_{0}\right)\right| \leq C_{1} \omega\left(d_{z}\right),
$$

whence

$$
M_{f}(z) \leq C_{1} \omega\left(d_{z}\right), \quad z \in E .
$$

On the other hand, (ii) tells us that

$$
M_{f}(z)-|f(z)| \leq C_{1} \omega\left(d_{z}\right), \quad z \in \mathbf{B}_{n},
$$

and we combine this with the elementary inequality

$$
a \log \frac{b}{a} \leq b-a \quad(0<a<b)
$$

to deduce that

$$
|f(z)| \log \frac{M_{f}(z)}{|f(z)|} \leq C_{1} \omega\left(d_{z}\right), \quad z \in \mathbf{B}_{n} .
$$

Finally, (3.3) and (3.4) together imply (iii).

(iii) $\Longrightarrow$ (iv). This is obvious.

(iv) $\Longrightarrow$ (i). Let $z \in E$. From (iv) we know that

$$
M_{f}(z) \leq C \cdot \omega\left(d_{z}\right),
$$


with some fixed $C>0$, and we deduce (e.g., by applying the Cauchy formula to a suitable polydisk centered at $z$ and contained in $B_{z}$ ) that

$$
\left|\partial_{j} f(z)\right| \leq \text { const } \cdot \frac{\omega\left(d_{z}\right)}{d_{z}} \quad(j=1, \ldots, n) .
$$

Hence

$$
|\nabla f(z)| \leq \mathrm{const} \cdot \frac{\omega\left(d_{z}\right)}{d_{z}}
$$

possibly with another constant on the right.

Now assume that $z \in E^{c}\left(=\mathbf{B}_{n} \backslash E\right)$, so that $f$ has no zeros in $B_{z}$. The function

$$
g_{z}(w):=f\left(z+\frac{d_{z}}{2} w\right) / M_{f}(z), \quad w \in \mathbf{B}_{n}
$$

is then zero-free and bounded in modulus by 1 on $\mathbf{B}_{n}$. An application of Lemma 2.1 gives

or equivalently,

$$
\left|\nabla g_{z}(0)\right| \leq 2\left|g_{z}(0)\right| \log \frac{1}{\left|g_{z}(0)\right|}
$$

$$
\frac{d_{z}}{2}|\nabla f(z)| \leq 2|f(z)| \log \frac{M_{f}(z)}{|f(z)|} .
$$

By (iv), there is a $C>0$ such that

$$
|f(z)| \log \frac{M_{f}(z)}{|f(z)|} \leq C \cdot \omega\left(d_{z}\right)
$$

and combining this with (3.6) we arrive at (3.5), this time for $z \in E^{c}$. Thus (3.5) actually holds for all $z \in \mathbf{B}_{n}$, and the proof is complete.

\section{References}

[1] Ahlfors, L. V.: Conformal invariants: topics in geometric function theory. - McGraw-Hill Series in Higher Mathematics, McGraw-Hill, New York-Düsseldorf-Johannesburg, 1973.

[2] Bishop, C. J.: Bounded functions in the little Bloch space. - Pacific J. Math. 142, 1990, 209-225.

[3] Dyakonov, K. M.: Equivalent norms on Lipschitz-type spaces of holomorphic functions. Acta Math. 178, 1997, 143-167.

[4] Dyakonov, K. M.: Besov spaces and outer functions. - Michigan Math. J. 45, 1998, 143-157.

[5] Dyakonov, K. M.: Multiplicative structure in weighted BMOA spaces. - J. Anal. Math. 75, 1998, 85-104.

[6] Dyakonov, K. M.: Holomorphic functions and quasiconformal mappings with smooth moduli. - Adv. Math. 187, 2004, 146-172.

[7] Garnett, J. B.: Bounded analytic functions. Revised 1st edition. - Springer, New York, 2007.

[8] Pavlović, M.: On Dyakonov's paper "Equivalent norms on Lipschitz-type spaces of holomorphic functions". - Acta Math. 183, 1999, 141-143.

[9] Rudin, W.: Function theory in the unit ball of $\mathbf{C}^{n}$. Reprint of the 1980 edition. - Classics Math. Springer-Verlag, Berlin, 2008.

[10] Shirokov, N. A.: Ideals and factorization in algebras of analytic functions that are smooth up to the boundary. - Tr. Mat. Inst. Steklova 130, 1978, 196-222.

[11] Shirokov, N. A.: Analytic functions smooth up to the boundary. - Lecture Notes in Math. 1312, Springer-Verlag, Berlin, 1988.

Received 5 January 2016 • Accepted 22 January 2016 